The data by Watanabe and colleagues emphasize the importance of innate immunity in driving a chronic inflammatory disease. Targeting TLR2 signaling may therefore be a useful approach in the treatment of individuals with Crohn disease that carry the NOD2 mutation. Another outcome of this study is the possibility of using NOD2 ligands as anti-inflammatory agents. This may explain the anti-inflammatory effects of berculosis remains a worldwide scourge, certain Gram-positive bacteria used in the treatment of inflammatory bowel disease. Perhaps probiotic bacteria contain ligands for NOD2. Further work on the importance of NOD2, and indeed other NODs, as important anti- or proinflammatory players is likely to hasten progress toward a deeper understanding of what goes wrong in chronic inflammation and possibly point the way to new therapies.
1. Hugot, J.P. et al. Nature 411, 599-603 (2001)

2. Ogura, Y. et al. Nature 411, 503-606 (2001).

3. Watanabe, T. et al. Nat. Immunol. 5, 800-808 (2004)

4. Inohara, N. and Nunez, G. Nat. Rev. Immunol. 3, 371-382 (2003)

5. Ogura, Y. et al. J. Biol. Chem. 276, 4812-4818 (2001).

6. Inohara, N. et al. J. Biol. Chem. 278, 5509-5512 (2003).

7. Girardin, S.E. et al. J. Biol. Chem. 278, 8869-8872 (2003).

8. Girardin, S.E. et al. Science 300, 1584-1587 (2003)

9. Kobyashi, K. et al. Nature 416, 194-199 (2002).

\title{
Mutual attraction: Does it benefit the host or the bug?
}

\section{JoAnne L Flynn

\begin{abstract}
Granulomas are thought to be immunological barriers that effectively contain mycobacteria. This hypothesis is now challenged by data that show granulomas are dynamic entities permissive to bacterial latency and reinfection.
\end{abstract}

T killing 2 million people per year. Although effective treatments are available, the regimens are lengthy and involve multiple drugs. In addition, many people with tuberculosis have inadequate access to health care. The AIDS epidemic has only made the situation worse, because HIV infection increases the risk of tuberculosis. In this issue of Nature Immunology, Cosma et al. provide exciting data that challenge old ideas and stimulate the generation of new hypotheses regarding this devastating disease ${ }^{1}$. The authors demonstrate that granulomas formed in response to mycobacterial infection are dynamic and that there is communication between the site of infection and the cells that take up the newly infecting bacilli. These results have implications for reinfection and the level of resistance provided by previous mycobacterial infection.

Although tuberculosis is prevalent in many countries, most people infected with Mycobacterium tuberculosis never develop active disease. Instead, $90 \%$ of infected persons contain the bacterium in a structure composed of immune cells, called the granuloma, and are considered to be latently infected. Reactivation of latent M. tuberculosis infection results in active tuberculosis. Thus, those people with latent infection serve as a 'reservoir' for future outbreaks of

JoAnne L. Flynn is in the Department of Molecular Genetics and Biochemistry, University of Pittsburgh School of Medicine, Pittsburgh, Pennsylvania 15261, USA.

e-mail: joanne@pitt.edu tuberculosis. Elimination of tuberculosis is a daunting task, given that some estimates suggest as many as 2 billion people are latently infected with this bacterium and are at risk for reactivating tuberculosis.

Granulomas form in response to chronic antigenic stimulation and, at least in the case of a tuberculous granuloma, are composed of macrophages, dendritic cells, $\mathrm{T}$ cells, B cells and sometimes fibroblasts. The bacteria are contained in this immunological microenvironment and are prevented from spreading throughout the lung and the body. The pathological consequences of this chronic infection are also contained in a focal area, preventing widespread inflammation and damage throughout the lungs. It has long been accepted that the granuloma is essential for control of $M$. tuberculosis infection and that the immune response at the level of the granuloma can be effective (in the case of latent tuberculosis) or inadequate (in the case of active disease).

The nature of the granuloma remains somewhat of a mystery. The granuloma is most likely a dynamic structure. Immune cells migrate to the site of infection, presumably in response to a series of signals from infected macrophages (Fig. 1). The migrating cells stop when they reach the infected macrophages and interact with the infected macrophages and with each other. The culmination of this cellular migration to the site of infection is the formation of the granuloma, and the granuloma can continue to recruit cells until bacteria are killed or even beyond that time. Reactivation occurs in response to impaired immune responses, resulting in bacterial growth in the granuloma and beyond its limits, as well as destructive pathology and disease. This course of events suggests that the organism in the granuloma can sense changes in the local immune response or that it 'tests' the environment and begins to grow when conditions are permissive (for example, when $\mathrm{CD}^{+} \mathrm{T}$ cells are depleted). However, the mechanisms by which granulomas are formed and maintained are not clear, and the extent of communication with the environment surrounding the granuloma is unknown. Cosma et al. now provide a glimpse into the dynamic nature of the granuloma ${ }^{1}$. Their study, using Mycobacterium marinum $^{2}$, demonstrates that an existing granuloma containing bacteria is not closed off from the environment and in fact seems to be a 'magnet' for cells carrying bacteria from a new infection. Macrophages or dendritic cells that pick up M. marinum from a new infection migrate to existing granulomas, suggesting that mycobacterial infection can program phagocytes to respond to signals from within the granuloma. However, the new infection must be similar to the infection that resulted in the formation of the granuloma initially. Infection of M. marinum-infected fish with salmonella did not result in targeting of the salmonella to the mycobacterial granulomas, even when the secondary infection was a mixture of mycobacteria and salmonella.

These data suggest that mycobacterial infection of macrophages or dendritic cells (the likely carriers of bacteria into the granulomas) induces a particular 'signature' that targets the cell to the signals 


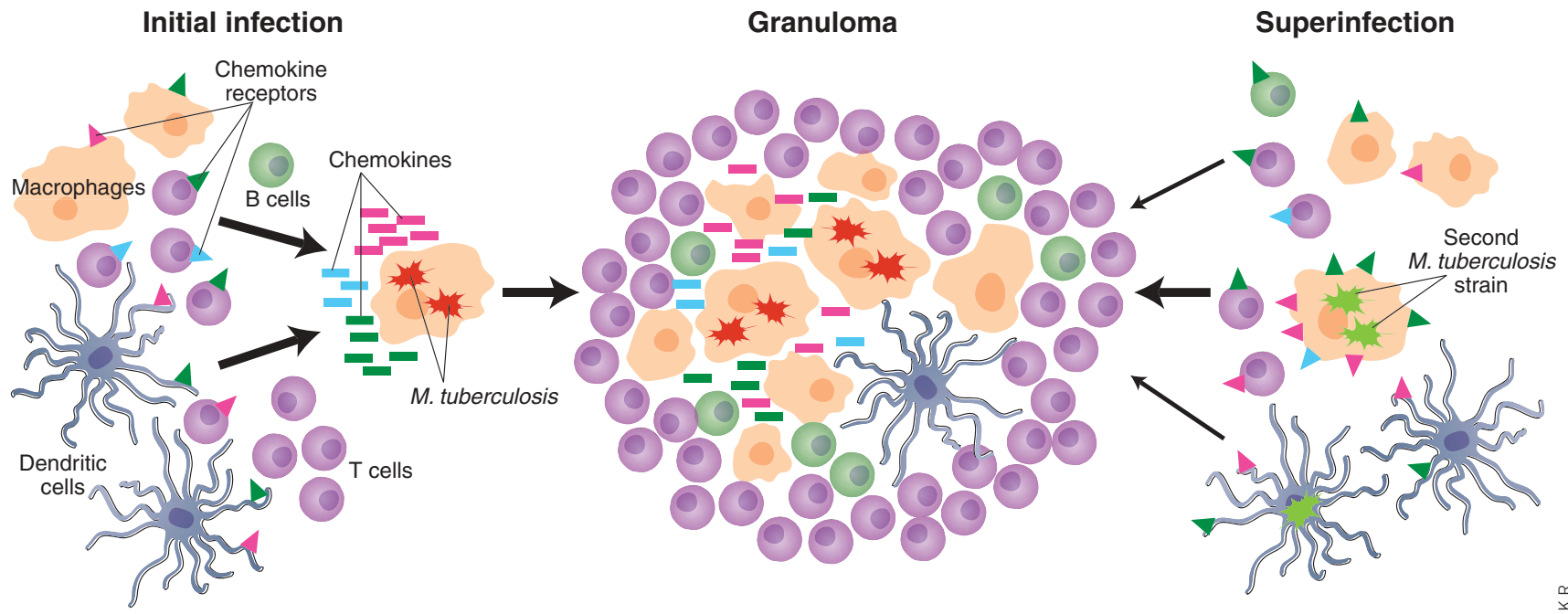

Figure 1 Formation of the granuloma and homing of bacteria into existing granulomas. Macrophages, dendritic cells, T cells and B cells express a variety of chemokine receptors that can respond to the chemokines expressed by infected cells in the granuloma. This results in migration of these cells to the site of infection. After migration, the cells remain at the site of infection, probably in response to a variety of signals expressed in the granuloma. When the host is reinfected, the phagocyte that ingests the organism may upregulate the appropriate chemokine receptors to a level even greater than that in uninfected cells, causing enhanced migration directly into the granuloma.

expressed by M. marinum-infected cells in the granuloma. This signature may be chemokine receptors that can sense specific chemokines expressed by mycobacteriainfected cells ${ }^{3}$ (Fig.1). Salmonella, in contrast, probably programs the macrophages or dendritic cells to respond to a different set of signals (for example, by expressing a unique combination of chemokine receptors), and therefore these cells would not migrate toward a mycobacterial granuloma. This process is likely to be important in the initiation of the infection and formation of the granuloma, as well as during an ongoing infection, during which any 'escaped' mycobacteria outside the granuloma should be targeted to a granuloma for containment. As a 'bonus' for the host, newly infecting bacteria are probably best contained by a pre-existing immune structure, and therefore targeting the bacteria to the granuloma makes sense for the host. Future studies in this model or other models may concentrate on defining the type of cell that is infected and targeted to the granuloma. The rapid modulation of a cell by a bacterial infection to respond to signals for migration is of considerable interest. Both the molecules induced on the dendritic cell or macrophage as well as those signals produced by the granuloma and the distance over which these signals can function are ripe areas for future studies. Comparison of cells infected in vivo with mycobacteria versus salmonella as well as careful analysis of molecules induced in the granuloma may provide some clues into what directs cells at the site of infection.

The repertoire of vaccines available at present against infectious diseases is based on the mechanistic understanding that the immune system responds with heightened efficiency to previously encountered pathogens. However, Cosma et al. show that targeting of newly introduced mycobacteria to a pre-existing granuloma does not seem to result in improved elimination of the organism. This observation supports the idea of the potential for previously (and latently) infected people to be reinfected with $M$. tuberculosis, indicating that prior infection does not protect against new infections. Although it is widely believed that a person who has positive results with the tuberculin skin test because of previous infection has a lower risk of developing active tuberculosis after new exposure to the organism than do people who have negative results", the advent of 'molecular fingerprinting' of strains provides ample evidence that reinfection can and does result in active cases of tuberculosis, even in areas of low endemicity ${ }^{5,6}$. Patients with active tuberculosis can have more than one strain present ${ }^{7}$, and effective treatment of active tuberculosis cases does not necessarily prevent reinfection and subsequent tuberculosis ${ }^{8}$. In addition, it is impossible to determine how often reinfection of latently infected persons occurs, because in the absence of active disease there are no markers for reinfection; it is likely to be a common phenomenon, particularly in regions of endemic tuberculosis.

The prevalence of reinfection leading to active tuberculosis as well as the apparent inability of an established $\mathrm{T}$ cell response and granuloma to eliminate incoming organisms in the fish model (and in other models of tuberculosis ${ }^{9}$ ) do not bode well for development of vaccines that protect against infection with $M$. tuberculosis. However, if a vaccine could greatly improve the granulomatous environment, in terms of effector function against $M$. tuberculosis, the inherent targeting of organisms to this specialized and dynamic immune site, demonstrated in the fish and frog model, could allow better containment, a lower risk of developing disease and less reactivation of infection. Although not a perfect solution, at least in the near future, this might be a feasible goal for vaccine development against this devastating disease.

1. Cosma, C.L. et al. Nat. Immunol. 5, 828-835 (2004).

2. Ramakrishnan, L., N.A. Federspeil, S. Science 288, 1436-1439 (2000).

3. Algood, H.M., Chan, J. \& Flynn, J.L. Cytokine Growth Factor Rev. 14, 467-477 (2003).

4. Myers, J., Bearman, J. \& Botkins, A. Dis. Chest $\mathbf{5 0}$ 120-132 (1966).

5. Garcia de Viedma, D. et al. Arch. Intern. Med. 162, 1873-1879 (2002).

6. Lambert, M.L. et al. Lancet Infect. Dis 3, 282-287 (2003).

7. Warren, R.M. et al. Am. J. Respir. Crit. Care Med. 169, 610-614 (2004).

8. van Helden, P.D. IUBMB Life 53, 219-223 (2002).

9. Repique, C.J., Li, A., Collins, F.M. \& Morris, S.L. Infect. Immun. 70, 3318-3323 (2002). 